\title{
Receiver Characterization using Periodic Small-Signal Analysis
}

\author{
Ricardo Telichevesky and Ken Kundert \\ Cadence Design Systems, San Jose California \\ Jacob White \\ Massachusetts Institute of Technology, Cambridge Massachusetts
}

\begin{abstract}
Periodic small-signal analysis is a recently developed approach for AC and noise analysis of linear periodically-varying circuits. This paper presents several new approaches to characterizing the transfer functions, noise, and distortion of communication circuits using this new technique. A receiver is used as an example, but the techniques presented are applicable to a wide variety of circuits, including mixers, samplers, sample-and-holds, chopper-stabilized amplifiers, frequency multipliers and switched-capacitor filters.
\end{abstract}

\section{INTRODUCTION}

The increasing demand for low-cost mobile communication systems has greatly expanded the need for simulation algorithms that are both efficient and accurate when applied to RF communication circuits such as mixers. The difficulty is that RF circuits often process signals that contain closely-spaced large frequencies. For example, a typical cellular phone service consists of $10 \mathrm{kHz}$ voiceband channels near $900 \mathrm{MHz}$. A SPICE transient analysis is extremely expensive for circuits that contain such signals because it needs to take very small time steps to follow the $900 \mathrm{MHz}$ carrier, and simulate for a long time to adequately resolve the voiceband modulation. However, circuits such as amplifiers can be linearized about their DC operating point and a small-signal analysis such as AC analysis used to efficiently determine the sinusoidal steady-state response of the circuit over an arbitrarily range of frequencies. The same is not true of circuits such as mixers or switched-capacitor filters that must be driven with an additional large periodic stimulus in order to operate properly.

\section{Periodic Small-Signal Analysis}

A periodic small-signal analysis computes the steadystate response of a linear periodically time-varying circuit to a sinusoidal stimulus. The linear time-varying circuit is usually derived by linearizing some periodically-driven nonlinear circuit. PAC (periodic AC), PXF (periodic transfer

Published in May 1996 at the Custom Integrated Circuits Conference. Manuscript received December 8, 1995.

E-mail addresses: ricardo@cadence.com, kundert@cadence.com, and white@rle-vlsi.mit.edu. function), and PNoise (periodic noise) are periodic smallsignal analyses that can be viewed as the natural extensions of AC, XF, and Noise analyses to periodic circuits.

Periodic small-signal analyses address periodically-driven circuits using a two-step process. First, the periodic operating point is found by performing a periodic steady-state (PSS) analysis with only the large periodic stimulus applied. For the case of a mixer, typically only the LO is applied; with switched-capacitor filters, typically only the clock is applied. The circuit is then linearized about the periodic operating point, and the steady-state response of the periodically-varying linear circuit is computed assuming that it is driven with a small sinusoid at an arbitrary frequency. The time required to compute the response of the linearized circuit is about the same as needed to compute the periodic steady-state response to the large stimulus alone, regardless of the frequency of the sinusoid.

An important aspect of periodically-driven circuits such as mixers and switched-capacitor filters is that they exhibit frequency translation. Mixers are actually designed to exhibit frequency translation, whereas with SC filters it is inadvertent. Conventional small-signal analyses (AC and noise) linearize about the DC operating point to create a linear time-invariant representation of the circuit, which cannot exhibit frequency conversion effects. With the periodic small-signal analyses, the circuits are linearized about a time-varying operating point and so can exhibit frequency conversion effects. Thus the PAC and PNoise analyses are similar to SPICE's AC and noise analyses, except that they can be applied to periodic circuits where frequency translation plays a critical role. Examples include conversion gain in mixers, noise in oscillators and switched-capacitor filters, etc.

The original approach to periodic small-signal analysis used Gaussian elimination to factor and solve the set of $N$ dense linear equations that describe the periodicallyvarying linearized circuit at a particular frequency where $N$ is the number of circuit nodes [1],[2]. The time required to factor a dense $N \times N$ matrix is on the order of $N^{3}$, which limits this approach to circuits containing at most a few hundred nodes. However, by applying matrix-implicit Krylov-subspace techniques, the time required to solve the system of equations can be reduced to the order of $N$, allowing circuits with up to 5000 nodes to be simulated over a broad range of frequencies in a few hours [3], [4]. 
Since the periodic small-signal analyses are performed on a linear representation of the circuit, the computed response will be a linear function of the stimulus, regardless of the size of the stimulus. Clearly in the real circuit, the input must be small enough to avoid violating the assumptions of the small-signal analysis. However, once the circuit has been linearized, the amplitude chosen for the 'small' stimuli is arbitrary. Typically the stimulus is given an amplitude of one and a phase of zero to allow transfer functions to be computed directly. In this regard, the periodic small-signal analyses are similar to conventional small-signal analyses.

\section{A. Periodic AC Analysis}

The Periodic AC (PAC) analysis computes the response of a linear periodically-varying circuit to a small sinusoidal input at an arbitrary frequency. PAC is used to compute transfer functions for circuits that exhibit frequency translation. Such circuits include mixers, switched-capacitor filters, samplers, sample-and-holds, chopper-stabilized amplifiers, and the like.

When applying a small sinusoid to a linear time-invariant circuit, the steady-state response is a sinusoid at the same frequency. However, when applying a small sinusoid to a linear periodically time-varying circuit, the circuit responds with sinusoids at many frequencies, as shown in Figure 1. Thus, in a sense, PAC computes a series of transfer functions, one for each harmonic of the LO. However, with these transfer functions the input and output frequencies are offset by the frequency of the harmonic. The transfer functions are identified by labeling them with the offset from the input signal in multiples of the LO fundamental frequency. This is the same label that is used to identify the particular sideband of the output signal. For circuits where the input and output are at the same frequency, such as switched-capacitor filters, the desired transfer function or sideband is labeled 0 . For down-conversion mixers, the desired transfer function or sideband is labeled -1 because the output frequency is offset from the input frequency by -1 times the LO frequency. For up-conversion mixers, the desired transfer function or sideband is labeled +1 . For samplers, sidebands far from zero might be used. In Figure 1 , all transfer functions from $-\mathbf{3}$ to $+\mathbf{3}$ are computed.

The small-signal frequency $\left(f_{\text {in }}\right)$ can be arbitrarily close, or even equal to, the LO frequency $\left(f_{\mathrm{LO}}\right)$ with no performance penalty.

\section{B. Measuring Intermodulation Distortion using PAC}

A important use of PAC analysis is to measure the intermodulation distortion of amplifiers and mixers. The traditional approach to measuring intermodulation distortion is to apply two large closely spaced tones to the input and measure the third-order intermodulation products. Applying this approach with a circuit simulator is expensive because two tones that are closely spaced in frequency require

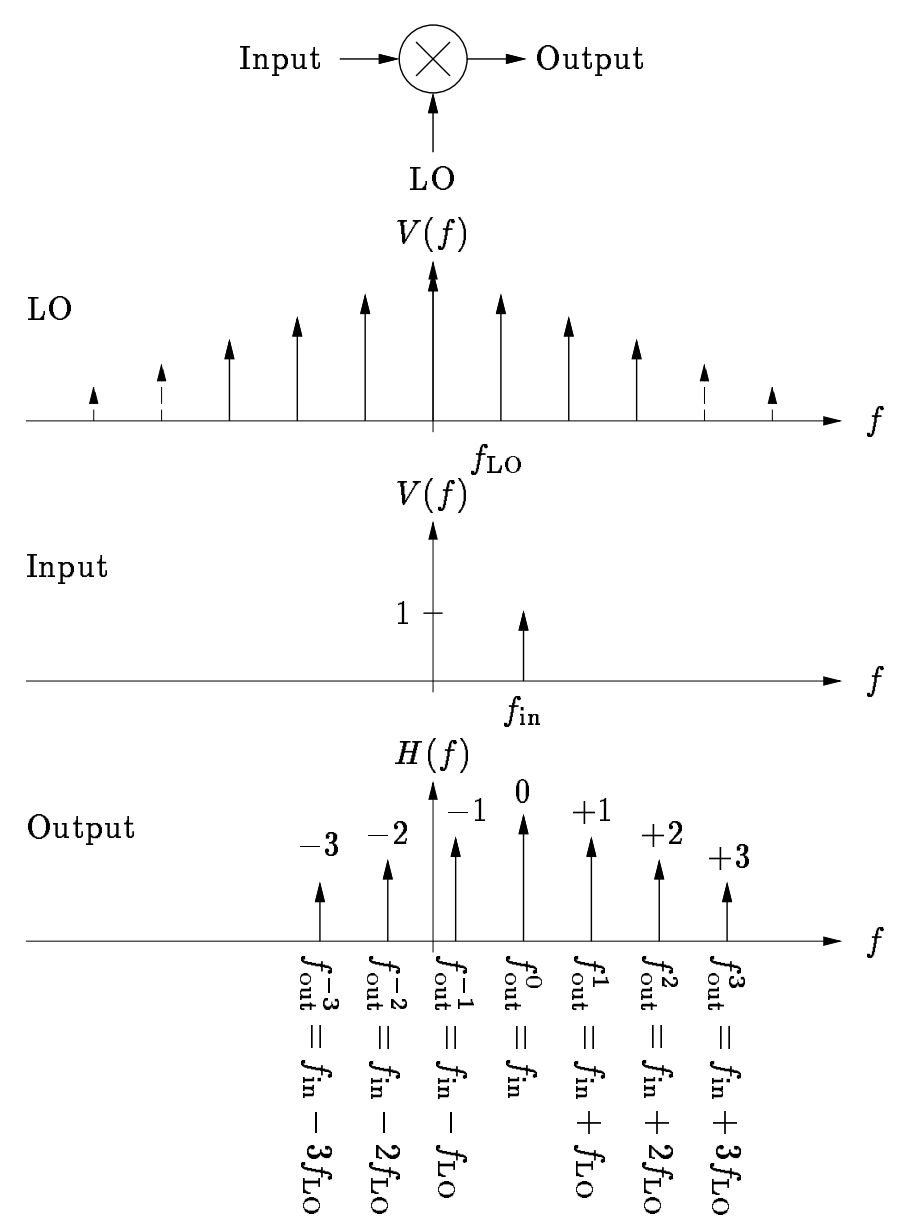

Fig. 1. The small-signal response of a mixer as computed by PAC analysis. The input signal is replicated and translated by each harmonic of the LO. In down-conversion mixers, the -1 sideband usually represents the IF output.

a long analysis interval. The approach used with PSS/PAC is slightly different. Here only one large tone is applied. The PSS analysis is performed with only that one large tone applied, and so is typically quite fast. Once the response of the circuit to the one large tone is computed, then PAC is used to apply the second tone close to the first. If the small input signal is considered to be one sideband of the large input signal, then the response at the other sideband is the third-order intermodulation distortion, as shown in Figure 2. In this case, the first tone (the tone applied in PSS analysis) drives the circuit hard enough to cause distortion and the second tone (the one applied in PAC) is a test tone that is only used to measure the intermodulation distortion. Once $V_{L 1}, V_{S 1}$, and $V_{S 3}$ are measured in decibels,${ }^{1}$ the third-order intercept point is

$$
I P_{3}=V_{L 1}-\frac{V_{S 3}-V_{S 1}}{2}
$$

\footnotetext{
${ }^{1} V_{L 1}, V_{S 1}$, and $V_{S 3}$ must be given in some form of decibels, however you are free to use your favorite form of decibels. The intercept point is computed in whatever form you choose.
} 


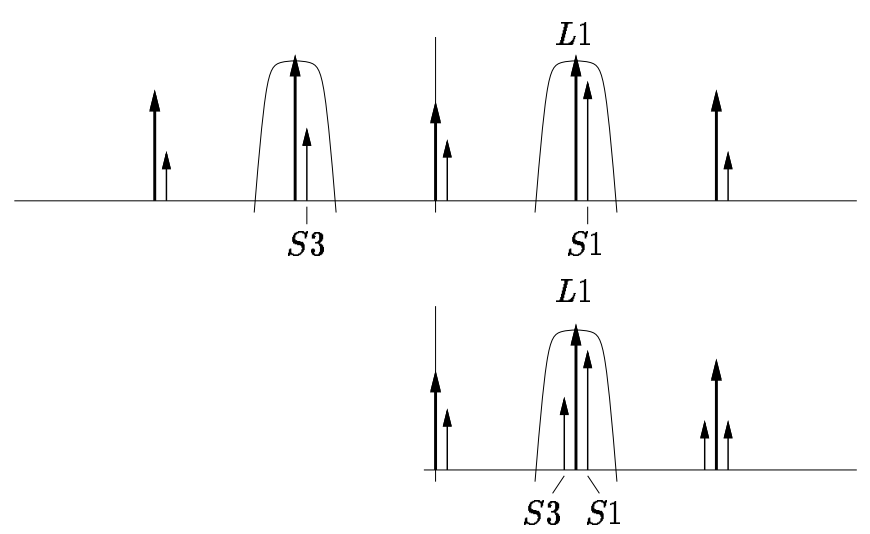

Fig. 2. Intermodulation distortion is efficiently measured by applying one large tone ( $L 1)$, performing a PSS analysis, and then applying the second small tone $(S 1)$ with a PAC analysis. In this figure, $V_{L 1}$ is the fundamental of the response due to the large input tone, $V_{S 1}$ is the fundamental of the response due to the small input tone and is the upper sideband of $V_{L 1} \cdot V_{S 3}$ is the lower sideband of $V_{L 1}$, and represents the intermodulation distortion. In the lower figure, all of the signals are mapped into positive frequencies, which is the more common way of viewing such results.

The intermodulation distortion of a mixer is measured in a similar manner, except the PSS analysis must include both the $\mathrm{LO}$ and the one large tone.

This approach gives slightly different results than the approach typically used when measuring intermodulation distortion on the bench. There, two large tones of equal amplitude are applied, both of which drive the circuit to behave nonlinearly. In this new method, only one tone is large enough to drive the circuit to behave nonlinearly. Both approaches are measuring intermodulation distortion; they simply use different ways of quantifying the results. Typically, when distortion levels are small, which they must be when computing $I P_{3}$ using (1), then the two methods produce results that are within one or two decibels. To mimic the PSS/PAC approach on the bench, simply reduce the amplitude of one of the tones.

Besides dramatically reducing the simulation time and memory, the PSS/PAC approach has other intesting benefits. If intermodulation distortion is measured using two large tones, then two intermodulation tones are produced and they are usually slightly different. A difference in amplitude in the intermodulation products usually indicates that the amplitude response of the circuit as a function of frequency is not flat at the measurement frequency. (It might also indicate that the two tones are not precisely the same size). On the bench, one can make the two test tones closer in frequency to reduce this effect, but this approach dramatically increases the time required for simulation. With the PSS/PAC approach, the separation between the tones can be arbitrarily small or they can be even at the same frequency. In this case, the ambiguity is resolved by accessing the signals by sideband index, rather than frequency, enabling accurate measurement of intermodulation distortion even where the frequency response is not flat,

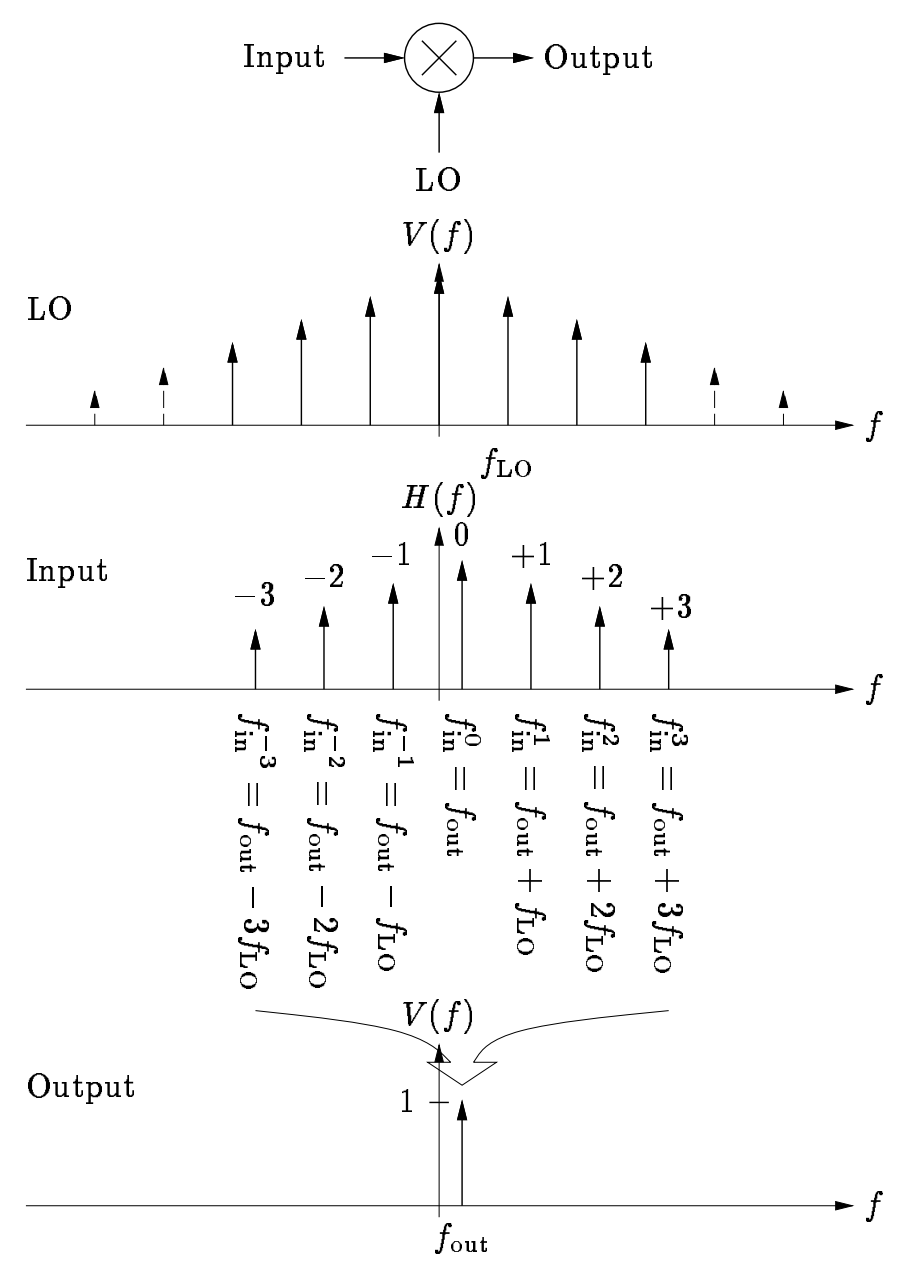

Fig. 3. The transfer functions computed by PXF analysis. The output is sensitive to signals at many frequencies at the input of the mixer. The input signals are replicated and translated by each harmonic of the LO. The ones shown are the ones that end up at the output frequency of interest.

such as along the skirt of a filter.

\section{Periodic Transfer Function Analysis}

A periodic transfer function (PXF) analysis computes the transfer function from every source in the circuit at any frequency to a single output at a single frequency. It differs from a PAC analysis in that the PAC analysis computes the response from a single stimulus at a single frequency to every node in the circuit at every frequency. This is illustrated in Figure 3. Generally, PAC is used when several transfer functions are needed that share the same input, and PXF is used when several transfer functions are needed that share the same output. The PXF analysis can simultaneously compute such useful quantities as conversion efficiency (transfer function from input to output at desired frequency), image and sideband rejection (input to output at undesired frequency), and LO feed-through and power supply rejection (undesired input to output at all frequencies). Frequency translation is taken into account 
in these quantities, so it is possible to determine how sensitive the output is to noise either up or down-converted from the supplies or the LO.

\section{Periodic Noise Analysis}

The periodic noise (PNoise) analysis is similar to the conventional noise analysis, except that it includes frequency conversion effects. Hence is it useful for computing the noise behavior of mixers, switched-capacitor filters, chopper-stabilized amplifiers, and the like.

PNoise is a two step process where PSS is used to compute the response to a large periodic signal such as a clock or LO. Results are labeled LO and shown in Figure 4. In the second step, which is the actual PNoise analysis, the resulting noise performance is computed. With periodic systems, there are two effects that act to translate noise in frequency. First, for noise sources that are bias dependent, such as shot noise sources, the time-varying operating point acts to modulate the noise sources. Second, the transfer function from the noise source to the output is also periodically time-varying and so acts to modulate the contribution of the noise source to the output. Modulation is a multiplicative effect in the time-domain and so in the frequency-domain the spectrum of the noise source is convolved with the spectrum of the transfer function. The transfer function is periodic and so has a discrete line spectrum. Convolution with a discrete spectrum involves a countable number of scale, translate, and sum operations, in a process known as noise folding, as shown in the rest of Figure 4. The final result of the analysis is the sum of the noise contributions both up-converted (negative labels) and down-converted (positive labels) to the desired output frequency.

\section{iII. Characterizing the Receiver}

The receiver being characterized is a rather large RF circuit designed by Tomas Melander of Ericsson Radio, and is shown in Figure 5. It is an image rejection receiver that consists of a low-noise amplifier, a splitting network, two double-balanced mixers, and broad-band Hilbert transform output filter combined with a summing network that is used to suppress the undesired sideband. It contains 167 bipolar transistors and uses 378 nodes. It is being simulated at the circuit level using the Gummel-Poon model for each transistor. It generated 986 equations (due to the large number of inductors and the BJTs parasitic resistors).

\section{A. Conversion Gain}

PXF analysis is performed to compute the conversion gain and the results are shown in Figure 6. Several transfer functions are shown. All are transfer functions from the RF input to the IF output, however each represents a different sideband. Unlike traditional transfer functions as computed by $\mathrm{AC}$ analysis, these transfer functions may have inputs and outputs at different frequencies. The output frequency range for all of the transfer functions shown is baseband, which is being swept from $20 \mathrm{kHz}$ to $200 \mathrm{MHz}$. For example, $V_{\mathrm{RF}}(1)$ is the transfer function from the first upper sideband $(780.02 \mathrm{MHz}-980 \mathrm{MHz})$ to baseband $(20 \mathrm{kHz}-200 \mathrm{MHz})$, and $V_{\mathrm{RF}}(-1)$ is the transfer function from the first lower sideband (580 $\mathrm{MHz}-797.98 \mathrm{MHz})$ to baseband. $V_{\mathrm{RF}}(2)$ is the transfer function from the second upper sideband to baseband, and finally, $V_{\mathrm{RF}}(0)$ is the transfer function from baseband to baseband.

Consider the dip at $700 \mathrm{MHz}$ in $V_{\mathrm{RF}}(-1)$, at which point the receiver exhibits $28 \mathrm{~dB}$ of attenuation. This implies that a signal from $V r f$ at $-700 \mathrm{MHz}$ mixes with the $-1^{\text {st }}$ harmonic of the LO and shows up attenuated at the output at $f_{\text {out }}=-700 \mathrm{MHz}+780 \mathrm{MHz}=80 \mathrm{MHz}$. Similarly, an $800 \mathrm{MHz}$ signal at the input would mix with the $1^{\text {st }}$ harmonic of the LO and would appear at the output at $f_{\text {out }}=800 \mathrm{MHz}-780 \mathrm{MHz}=20 \mathrm{MHz}$ amplified by $15 \mathrm{~dB}$. This strong asymmetry in the transfer function on either side of the LO is a clear indication that this receiver is designed to suppress the lower sideband.

Less than 4 minutes were required on an HP735/125 for this analysis, which contained 50 frequency points.

\section{B. Noise Figure}

The PNoise analysis is similar to the traditional noise analysis, except it includes the effect of noise moving from one frequency to another as it mixes with the LO and its harmonics. The noise measured at the output includes contributions from noise generated at frequencies $f_{\text {out }} \pm k f_{\mathrm{LO}}$, where $|k| \leq 7$, and 7 is an user setable parameter.

This noise analysis is performed on the detailed periodically varying linearized circuit, and so it includes a great deal of subtle effects in the calculation. For example, at the points in time where the LO signal is near $0 \mathrm{~V}$, the differential pairs that make up the mixer are balanced and so exhibit a great deal of gain from the LO to the output. Any noise on the LO is greatly magnified at this time. Once the LO moves away from 0 , the differential pairs saturate and so exhibit a great deal of attenuation from the LO to the output. Hence, the faster the LO moves through zero, the less noise is transferred from the LO to the output. This is why switching mixers are generally preferred over multiplying mixers. This effect is one of many that are accurately accounted for by the PNoise analysis.

The noise figure as computed by PNoise is shown in Figure 7 . Less than 4 minutes were required on an HP735/125 for 50 frequency points.

\section{Intermodulation Distortion}

The first step in computing intermodulation distortion is to configure PSS analysis to measure harmonic distortion. Thus, the LO and one large input tone are applied. 
Since the resulting signals must be periodic, the applied signals must be commensurate and have the smallest possible common period. In this circuit the $\mathrm{LO}$ is given as $780 \mathrm{MHz}$ and the input tone is chosen to be $840 \mathrm{MHz}$, which gives a $16.67 \mathrm{~ns}$ common period. The PSS analysis takes $2.5 \mathrm{~min}-$ utes and $93 \mathrm{MB}$ to compute the steady-state solution using 787 time-points. So far, only enough information is available to compute the receiver's harmonic distortion, which is useful in this case because this receiver is broadband. As a second step, a small $850 \mathrm{MHz}$ signal is applied and PAC analysis is run. Since only one frequency point is needed from the PAC analysis, it is very efficient, requiring only 47 seconds to compute the perturbation to the PSS solution due to the small $850 \mathrm{MHz}$ RF test signal.

$I P_{3}$ is computed using $V_{L 1}, V_{S 1}$, and $V_{S 3}$, where $V_{L 1}=$ $-25.7 \mathrm{dBV}$ is the magnitude at the output of first fundamental signal at $60 \mathrm{MHz}, V_{S 1}=2.6 \mathrm{dBV}$ is the magnitude at the output of the second fundamental signal at $70 \mathrm{MHz}$, and $V_{S 3}=-49.7 \mathrm{dBV}$ is the $I M_{3}$ term at the output at $50 \mathrm{MHz}$. The third-order intercept point computed using (1) is $0.45 \mathrm{dBV}$.

To confirm this result, a 3-tone PSS analysis was performed. The results were within $1 \mathrm{~dB}$, with the PSS/PAC approach requiring 6 times less time and memory. This ratio would be much greater if the receiver were followed by a narrow-band filter, and so required closer spacing of the intermodulation tones.

\section{CONCLUSION}

Conventional small-signal analyses, such as $\mathrm{AC}$ and noise analyses, are widely considered essential by those engineers involved in designing amplifiers and filters. Periodic smallsignal analyses provide these same measurement capabilities for periodically-driven circuits such as mixers, detectors, samplers, frequency multipliers, phase-locked loops, oscillators, parametric oscillators, switched-capacitor and switched-current filters, chopper-stabilized and parametric amplifiers, sample-and-holds, and etc. In addition, it is shown how to use a periodic small-signal analysis to efficiently measure intermodulation distortion. It is felt that these measurement capabilities will soon be considered as essential for periodically-driven circuits as $\mathrm{AC}$ and noise analysis are for amplifiers and filters.

\section{REFERENCES}

[1] M. Okumura, T. Sugawara, and H. Tanimoto. "An efficient small signal frequency analysis method for nonlinear circuits with two frequency excitations." IEEE Transactions of Computer-Aided Design of Integrated Circuits and Systems, vol. 9, no. 3, pp. 225235, March 1990.

[2] M. Okumura, H. Tanimoto, T. Itakura, and T. Sugawara. "Numerical Noise Analysis for Nonlinear Circuits with a Periodic Large Signal Excitation Including Cyclostationary Noise Sources." IEEE Transactions On Circuits and Systems - I Fundamental Theory and Applications., vol. 40, no. 9, pp. 581-590, September 1993.
[3] Ricardo Telichevesky, Kenneth S. Kundert, Jacob K. White. "Efficient Steady-State Analysis based on Matrix-Free KrylovSubspace Methods." 1995 Design Automation Conference.

[4] Ricardo Telichevesky, Kenneth S. Kundert, Jacob K. White. "Efficient AC and Noise Analysis of Two-Tone RF Circuits." Submitted to the 1996 Design Automation Conference. 


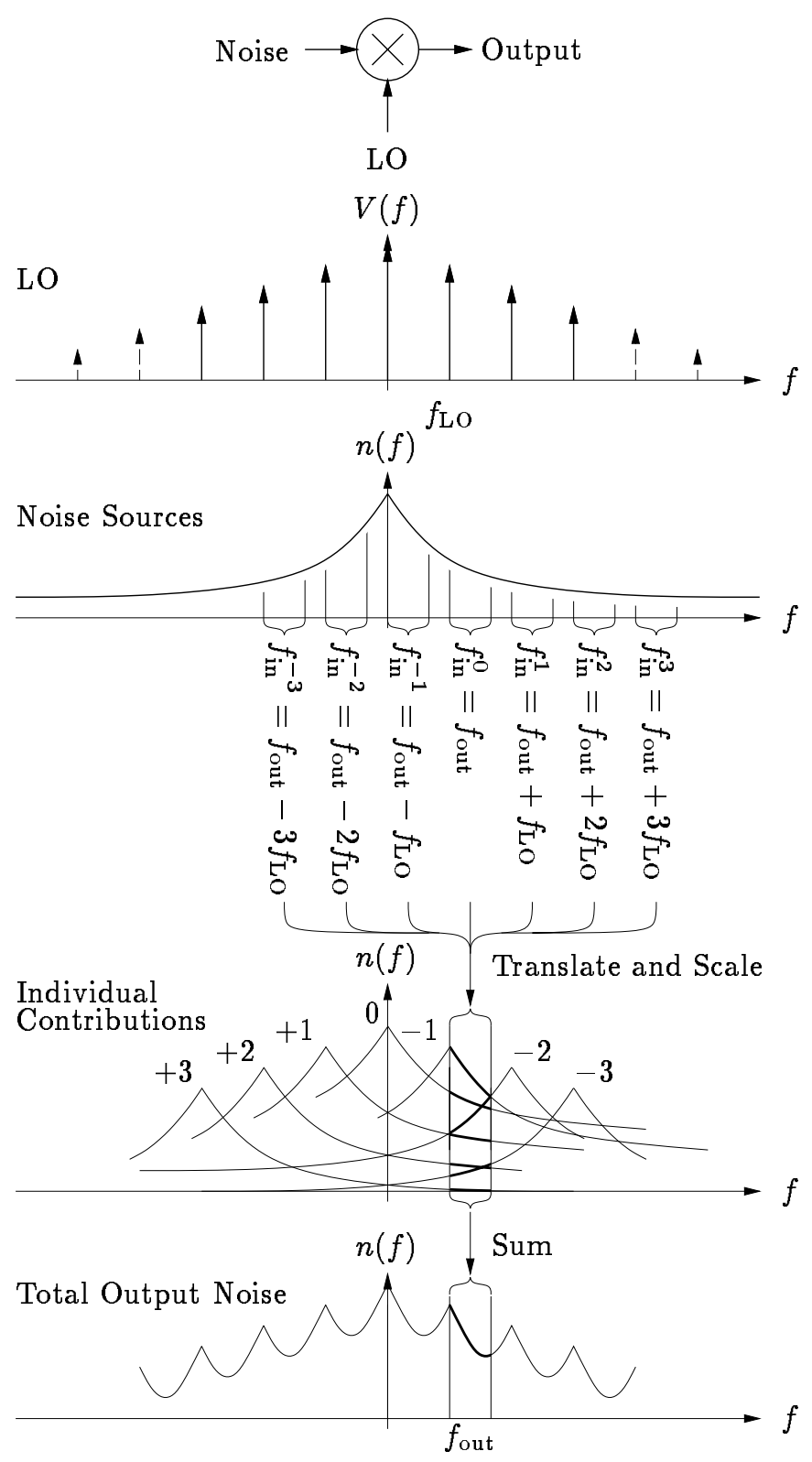

Fig. 4. How noise is moved around by a mixer. The noise is replicated and translated by each harmonic of the LO. The PNoise analysis computes the total noise including the modulation effects caused by the LO.

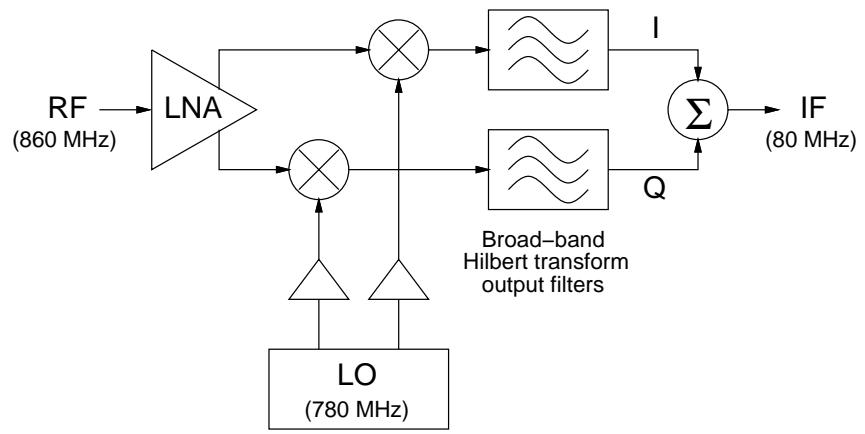

Fig. 5. The block diagram of the high-performance image-rejection receiver being characterized.

Transfer Functions from Vrf to V(out) over $20 \mathrm{kHz}$ to $200 \mathrm{MHz}$

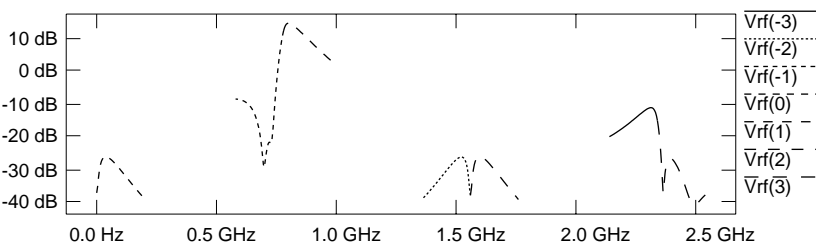

Transfer Functions from Vrf to V(out) over $20 \mathrm{kHz}$ to $200 \mathrm{MHz}$

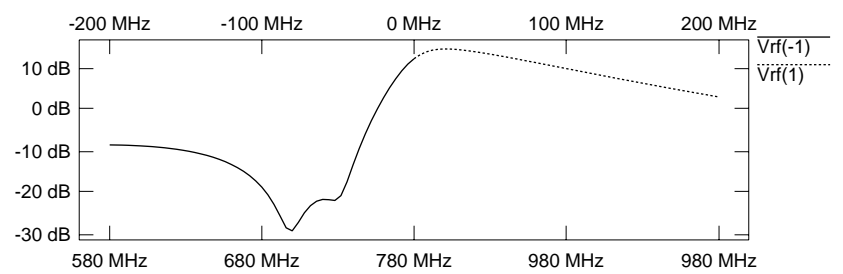

Fig. 6. The transfer functions from the $V_{r f}$ input to $V_{o u t}$. The bottom figure is an expanded version of the top that clearly shows the conversion due to the \pm 1 harmonics of the LO. The bottom axis is labeled with the input frequency, and the top axis is labeled with the output frequency.
Noise Figure from Vrf to V(out)

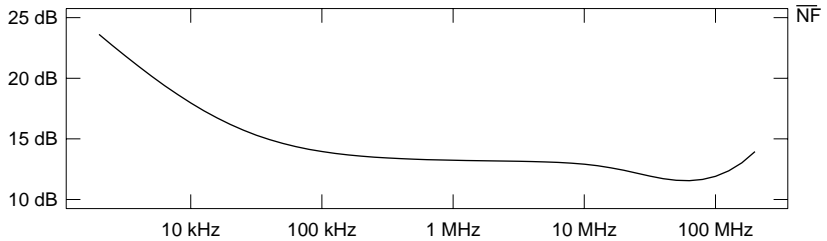

Fig. 7. The noise figure from $\operatorname{Vrf}$, assuming $50 \Omega$ source resistance. 